\title{
PERCEPCIONES DEL ENVEJECIMIENTO: GERDA MARSHALSON EN HENRY AND CATO (1976), DE IRIS MURDOCH
}

\section{PERCEPTIONS OF AGEING: GERDA MARSHALSON IN IRIS MURDOCH'S HENRY AND CATO (1976)}

\author{
Mariángel Solans García \\ Universidad Nacional de Educación a Distancia (UNED) \\ masolans@flog.uned.es
}

Fecha de recepción: 03-09-2020

Fecha de aceptación: 28-10-2020

\section{RESUMEN}

El presente artículo plantea una aproximación a la novela Henry and Cato (1976) de la escritora anglo-irlandesa Iris Murdoch, analizando desde la perspectiva de los estudios de la edad el modo en que se articulan en ella los significados culturales del envejecimiento. El análisis se centra en la codificación de la edad en el cuerpo de una de las protagonistas, Gerda Marshalson, las asimetrías en las relaciones de poder por razón de edad, y los comportamientos edadistas que refleja su entorno. De este modo no sólo se centra en los estereotipos relacionados con el complejo proceso del envejecimiento que la obra reproduce, sino también en la subversión de los mismos.

Palabras Clave: Iris Murdoch; Henry and Cato; envejecimiento; edad; género; empoderamiento

\section{ABSTRACT}

This paper proposes an approach to the novel Henry and Cato (1976) by the AngloIrish writer Iris Murdoch, analysing from an ageing studies perspective the way in which the cultural meanings of ageing are articulated in this text. The study deals with 
the codification of age in the body of one of the protagonists, Gerda Marshalson, the asymmetries in age power relations, and the ageist behaviours that her environment reflects. In this way, it focuses not only on the stereotypes related to the complex ageing process that the novel reproduces, but also on their subversion.

KeYwords: Iris Murdoch, Henry and Cato, ageing, age, gender, empowerment

\section{INTRODUCCIÓN}

Uno los mayores desafíos que afronta la población mundial en la actualidad es su progresiva longevidad. De acuerdo con Mike Hepworth (2000: 9) «ours is probably the most age-conscious period in human history». A esta preocupación demográfica y social hemos de añadir el fenómeno conocido como la «feminización del envejecimiento», que produce un desequilibrio entre sexos que se acentúa con la edad. La literatura, como foro de discursos, ofrece la oportunidad de ahondar en las distintas representaciones de este proceso de envejecimiento, una realidad multidimensional y compleja que atiende a cambios biológicos, psicológicos y sociales. Los estudios de la edad como materia literaria han tomado fuerza dentro de los estudios culturales, partiendo de la base de que la literatura no sólo refleja contenidos y actitudes que contribuyen a elaborar representaciones sociales, sino que, de manera inversa, también las construye. Esta investigación tiene como objetivo analizar cómo la escritora anglo-irlandesa Iris Murdoch representa el paso del tiempo en su novela Henry and Cato (1976), centrándonos en el personaje de Gerda Marshalson. De esta forma expondremos cómo Gerda negocia los significados del envejecimiento y la sumisión y resistencia que muestra ante un modelo edadista que su hijo trata de imponerle.

El pasado año 2019 celebramos el centenario del nacimiento de Iris Murdoch (19191999), una de las escritoras en lengua inglesa más influyentes y prolíficas de la segunda mitad del siglo XX. La dimensión de su legado incluye, entre otros, veintiséis novelas, dos volúmenes de poesía, una obra de teatro radiofónica y un libreto de ópera. A este corpus debemos agregar su faceta como filósofa, que incluye cinco volúmenes y numerosos ensayos. Henry and Cato pertenece a la etapa de madurez de la creación literaria de Murdoch, cuando sus obras son cada vez más extensas, originales y complejas. La crítica suele coincidir en destacar las novelas de este periodo como sus mejores aportaciones.

Además de dar título a esta novela, Henry y Cato son sus principales protagonistas, dos amigos de la infancia que con el paso de los años vuelven a encontrarse. De personalidades diferentes y al mismo tiempo complementarias, Henry Marshalson es un joven profesor de historia del arte en una universidad de Illinois; Cato Forbes, en cambio, es un sacerdote católico. Ambos responden a los modelos de personajes que Murdoch define como el existencialista y el místico (Sodre, 1976: 5). Henry y Cato vivieron su infancia y juventud en casas vecinas: Laxlinden Hall y Pennwood. Pertenecen a una clase social acomodada, especialmente Henry. Los Marshalson son una familia de terratenientes que, aun sin ostentar título aristocrático, poseen una mansión ancestral además de extensos terrenos. En Laxlinden habita la matriarca de la familia, Gerda Marshalson, acompañada de su amigo Lucius 
Lamb, un escritor venido a menos que ahora vive a su costa. A escasos metros de Laxlinden se encuentra Pennwood, la residencia de John Forbes, el padre de Cato y viudo al igual que Gerda. Ambos progenitores encarnan una actitud conservadora que se contrapone a la rebeldía de sus hijos.

La transición a lo largo de las etapas de la vida es uno de los temas claves de esta novela, ya sea el paso de Henry y Cato a otra fase de la adultez, o la transición de sus padres hacia la madurez. El acontecimiento que desencadenará un cambio en sus vidas, y les despertará del estado de limbo en el que viven, será su reencuentro tras el inesperado fallecimiento de Sandy, el hermano mayor de Henry y por tanto heredero de Laxlinden. ${ }^{1}$ Tras su muerte en un accidente de coche, la propiedad pasará a manos de Henry. Pero dentro de este traspaso juega un papel importante una mujer: Stephanie Whitehouse, a quien todos tienen por examante de Sandy cuando en realidad era su empleada doméstica. En contra de las expectativas de su madre, Henry pretende romper con el legado familiar deshaciéndose de la mansión y contraer matrimonio con Stephanie. En paralelo a esta trama, también se nos narran los acontecimientos en la vida de su amigo Cato Forbes, quien en contra de las expectativas de su padre, se ordena sacerdote. Sin embargo, su vocación se desmorona cuando se enamora de un joven delincuente que acude a su parroquia y al que mata accidentalmente. Henry finalmente logrará reconciliarse con su pasado y, cediendo a los deseos de su madre, reconducirá su vida junto a Colette Forbes, la hermana pequeña de Cato.

\section{GERDA: LA AUTORIDAD DE LA EDAD Y LA TRADICIÓN}

En el presente artículo nos hemos propuesto reflexionar sobre el modo en que Gerda Marshalson responde al paso del tiempo y negocia los significados de la edad. Gerda es una mujer de carácter solitario que se nos muestra tras un muro de frialdad, incapaz de exteriorizar sus sentimientos. Las connotaciones jerárquicas y autoritarias que denota su apellido (marshal) describen la esencia de su personalidad. La identidad de Gerda ha estado definida dentro de la familia por los roles tradicionales que le imponen la sociedad patriarcal y el sistema patrimonial. Ha sacrificado su vida dedicándola al cuidado de los hombres con los que ha vivido (su marido e hijos) y a la hacienda familiar, acatando formalmente las normas impuestas.

Ateniéndonos a cuestiones socioculturales, la figura de la madre tiene poco peso en una familia en la que el patrimonio se transmite por vía masculina y su papel queda asociado a su función reproductiva. Por ello, el bienestar económico de Gerda se ha basado en un modelo tradicional de dependencia: primero de su marido, Burke Marshalson y a su muerte, siguiendo la norma primogeniturista, de su hijo Sandy, heredero de todos los bienes familiares. Su viudedad, por tanto, se traduce en una disminución de su renta y de estatus social.

Las muertes de su marido, y más tarde la de su hijo Sandy, marcan momentos de discontinuidad en su vida y la arrojan a una soledad que le resulta aterradora, pese a haber sido

\footnotetext{
1 La misma trama en la que un fallecimiento provoca un reencuentro familiar aparece en otras novelas de Murdoch, como An Unofficial Rose (1962) o The Italian Girl (1964).
} 
una mujer solitaria desde su infancia como hija única. Tras la muerte de Sandy, Gerda se enfrenta a una nueva vida en una casa en la que «her wandering feet roused echoes which she had never heard before» (Murdoch, I976: 37). Su aislamiento social, confinada en una gran casa rural cercada por una tapia, alejada del pueblo más cercano y rodeada de naturaleza, también contribuye a su sensación de soledad. Su propio hijo es consciente de la soledad de su madre cuando afirma que «I suppose that women [...] learn pretty early on that they've got to be alone and bear things alone, even when they're in the bosom of their family» (Murdoch, 1976: 196). La única compañía de Gerda es la de su sirvienta Rhoda y la de Lucius Lamb, ${ }^{2}$ un poeta al que en otro tiempo amó, pero en quien ahora sólo busca alguien que la acompañe y que la siga admirando. Por lo tanto, de acuerdo con el sociólogo Robert Weiss (en Friedan, 2006: 397), en Gerda se encuentran dos tipos de soledad: una de tipo emocional y otra de tipo social, ya que carece de comunidad o amigos a los que sentirse unida, un hecho habitual entre los personajes femeninos de Murdoch.

Las apariciones de Gerda tienen lugar dentro del recinto de la casa, bien en el exterior mientras se dedica a las labores de jardinería, o como suele ocurrir, en su interior, retirada de la vida social. Solemos localizarla contemplando el mundo a través de las ventanas o bien sentada junto a la chimenea, cuya llama reaviva constantemente en un intento de evitar su extinción. El fuego que alumbra la biblioteca recuerda el mito de la caverna de Platón, tan presente siempre en la narrativa de Murdoch, sugiriendo una existencia en un mundo de apariencias, encadenada a un modo de vida y una época que se extingue y que ya no le aporta nada.

Al comienzo de la obra encontramos a Gerda preocupada por la llegada de su hijo Henry, que regresa de Estados Unidos como heredero de su hermano, y con el que nunca ha mantenido una buena relación. Madre e hijo representan dos continentes asociados a valores contrapuestos: la «vieja» Europa y la «joven» América. Esta caracterización alegórica permite abordar el tema de la representación de la edad a través de las dos generaciones de Marshalson. Por un lado, Henry es el hijo soltero que apuesta por el modelo liberal estadounidense y que vuelve a su casa inglesa, ahora venida a menos tras haber vivido durante nueve años en Estados Unidos, la tierra de la libertad y la inocencia ${ }^{3}$, Henry encuentra Europa claustrofóbica y apuesta por el sueño americano. Gerda, en cambio, representa los valores rancios y trasnochados de la sociedad británica. Como representante de la tradición europea, Gerda odia América, considerándola «raw, ugly, vulgar, frightening and curiously empty» (МuRDOCH, 1976: 323).

\section{LA LECTURA DEL CUERPO DE GERDA}

El cuerpo como discurso se articula como un texto en el que se leen los signos del paso del tiempo y las distintas lecturas que en él se inscriben. Por ello analizaremos el modo en

\footnotetext{
2 Su apellido podría aludir al ensayista y poeta británico Charles Lamb, quien al igual que Lucius, nunca alcanzó éxito como poeta.

3 La oposición entre la joven América y la vieja Europa también aparece en The Sea, the Sea (Murdoch, 1978) a través de la confrontación de valores entre la madre del protagonista y su tía norteamericana o en A Fairly Honourable Defeat (Murdoch, 1970), representada por el personaje de Julius King.
} 
que Gerda percibe esta metamorfosis y la sensación de otredad que experimenta en esta etapa de su vida. De acuerdo con Hepworth, «age identity emerges out of interaction between younger and older people» (2000: 63). Así ocurre en el caso de Gerda, quien obtiene el reflejo de su envejecimiento en la mirada de la generación más joven: su hijo Henry y la joven Colette. Este hecho evidencia tanto el conflicto entre generaciones como la cualidad relacional de la edad.

A su llegada a la casa, el joven Henry contempla a través del ventanal a su madre, que se encuentra en el interior. La gran ventana funciona como una pantalla a través de la cual Henry, como espectador pasivo, advierte sorprendido cómo se ha inscrito el paso del tiempo en el cuerpo de su madre tras casi diez años sin haberse visto (también en el propio Henry, aunque la pantalla no refleje su propia imagen). El recuerdo congelado de su madre choca con la realidad, experimentando una fuerte impresión al escrutar su apariencia. El rostro de Gerda se muestra tal cual es, sin enmascarar su edad tras capas de maquillaje, ni pretender pasar por alguien más joven. Su cuerpo permanece invisible bajo largas y amplias túnicas de distintos colores. Aunque Henry hubiera preferido encontrarla joven y guapa, conservada como un objeto que el tiempo no altera, descubre a una Gerda avejentada y más gruesa:

Her hair isn't grey, was his first thought, or else she dyes it. But her face was older, older; and in that shock of seeing her he realized for the first time how much he had somehow hoped that she would still look young and beautiful. She seemed thicker, her face fatter, coarser, though not lined. Her mouth looked larger, more masculine. (MURDoCH, 1976: 76)

La condición andrógina del envejecimiento biológico, causada por el descenso de ciertas hormonas merma la construcción ideal de la feminidad. El físico de Gerda también describe a una mujer fuerte psicológicamente y que se acepta tal como es: «older certainly, fatter, but still handsomely carrying that old air of a beauty's confidence» (MURDOCH, 1976: 86). También la mirada masculina de Lucius se posa sobre Gerda, sentenciando mientras la observa en casa: «of course she was faded and her features were less fine» (MuRdoch, 1976:10).

El envejecimiento físico de Gerda se define por tanto en la interacción con otras generaciones más jóvenes que se presentan como espejos que reflejan «the objective certainty of our transformation» (DE BEAUvoIR, 1996: 260). Uno de los momentos más representativos del confrontamiento de Gerda con su edad y la idea de la otredad que ello implica es cuando coincide con Colette Forbes, quien por su juventud se asocia a lo natural y lo reproductivo. Es en este encuentro cuando más apreciamos el conflicto entre su sentimiento subjetivo de juventud interna y el proceso externo de su envejecimiento biológico. Gerda alaba cómo a su edad, la joven y lozana Colette puede llevar cualquier prenda y lucir un aspecto maravilloso, a lo que Colette responde elogiando el estupendo aspecto físico de Gerda, aunque considerándolo excepcional y situando el esplendor de su belleza en el pasado: «I don't know if anyone ever told you - but you're, well, even now - someone must have told you when you were young» (Murdoch, 1976: 190). Las palabras de Colette despiertan un sentimiento de nostalgia que distancia a Gerda del momento actual, «that was a long time ago» (Murdoch, 1976:190). Esta experiencia subjetiva se percibe como «a moment of ageing» 
(ARIAS, 2005: 3), un momento en el que a través de la mirada externa, Gerda se hace consciente de su propio envejecimiento.

La lectura del cuerpo enfrenta a Gerda al canon de belleza, (re)productividad y eficacia que encarna la generación más joven y que despierta la conciencia del paso del tiempo. La atención que la sociedad occidental coloca en el físico de la mujer mayor que se desvía de los ideales de belleza vulnera la autoestima de Gerda, que reacciona huyendo tras escuchar las palabras de Colette. El choque de tiempos impide a Gerda recuperar su imagen pasada como mujer joven y bella, lo cual le produce dolor: «glancing into the driving mirror could see nothing because her eyes were full of tears» (MURDOCH, 1976: 190). Esta mirada retrospectiva reactiva un tiempo en el que contaba con «scores, hundreds, to laud her beauty» (MURDOCH, 1976:190); es decir, cuando aún respondía al ideal de belleza femenina que la sociedad impone a las mujeres. Esta pérdida, unida a las pérdidas afectivas que ha sufrido Gerda, convierte su envejecimiento en «a process of dispossession» (WoODWARD, 1991: 149).

El personaje de Gerda también refleja la idea de que a una determinada edad ya no hay tiempo para el amor y la sexualidad y, si ello ocurre, se percibe como un acontecimiento a destiempo. Gerda lo asume como un código de conducta impuesto por su edad, considerando escandaloso que Henry le cuestione si Lucius Lamb es su amante. Gerda, que ya no busca en Lucius el amor romántico ni sexual sino mera compañía, considera la pregunta una falta de respeto. Sin embargo, Henry, ostentando una posición de superioridad, piensa que su nuevo estatus como hombre y heredero de la mansión le da derecho a controlar la vida privada de su madre. La respuesta de Gerda («as if I'd have a lover at my age» (MuRDOCH, 1976: 219)) indica la interiorización y asimilación del estereotipo según el cual una edad madura descalifica sexualmente a la mujer (SonTAG, 1997: 20). Su imagen corporal ya no responde al canon de un cuerpo joven y potencialmente (re)productivo y, por tanto, deseable. Por ello, siguiendo un código de conducta propio de su edad, Gerda debe mantenerse al margen: «nothing is more distasteful than an elderly woman who acts young and wants to be in the limelight all the time» (Murdoch, 1976: 219). Dicho código no afecta, en cambio, a su vecino John Forbes, también viudo y mayor que ella, quien tiene una amante a la que visita frecuentemente en Londres. Sin embargo, es una relación mantenida en secreto ante sus hijos Cato y Colette, quienes podrían considerar escandaloso o irrisorio que dos personas que han superado la madurez tengan una aventura sentimental.

\section{LAXLINDEN COMO ALTER EGO DE GERDA}

El principal escenario de la novela, Laxlinden, funciona como una potente imagen que conecta el deterioro del espacio físico con el proceso de envejecimiento de sus ocupantes y nos permite además analizar el apego por los objetos del pasado.

La mansión de los Marshalson representa el alter ego de Gerda, símbolo de la tradición y de la conexión entre lo femenino y lo doméstico. Es el lugar sobre el que hasta ahora ejerce su autoridad, aunque legalmente nunca le haya pertenecido. Se trata de un espacio con el que está íntimamente familiarizada, cuyos muebles, adornos y todo lo que contiene refleja su manera de ser. Atendiendo a Friedan, la casa proporciona un sentimiento de con- 
tinuidad y unidad (2006: 363), como si se tratara de una prolongación de sí misma. Como testigo de la experiencia cotidiana, la casa familiar ayuda a Gerda a mantener su identidad y el sentido de sí misma ante las pérdidas que experimenta en su vida.

Las paredes de la casa están sobrecargadas de objetos acumulados a lo largo de los años. Los retratos antiguos que ocupan las paredes, entre ellos uno en el que aparece la mansión con catorce personas de servicio, funcionan como soporte físico de memoria, atestiguando el devenir de la economía familiar. A partir de un lenguaje meticulosamente descriptivo, Murdoch ofrece su visión de Laxlinden, que ahora presenta una tonalidad apagada, reflejo de la monotonía y la tristeza que reina en las vidas de sus inquilinos. En un acto simbiótico, cuerpos y espacios se unen en el envejecimiento (HEPWORTH, 2000: 72). En la casa prevalecen los tonos otoñales, amarillos y marrones, como metáfora del período de madurez en el que se encuentra Gerda. Los narcisos que evocan la juventud y la primavera ya se han cerrado y sus hojas comienzan a marchitarse. Observamos un tapiz polvoriento, estanterías con libros ancestrales que amarillean con el paso de los años, empapelados descoloridos, alfombras de lana gastadas, sillas rotas y sillones hundidos. De acuerdo con Hoskins, los muebles funcionarían como objetos biográficos, conectando su deterioro gradual con el propio proceso de envejecimiento de los habitantes de la casa (1998: 8).

En la sociedad tradicional británica se da por sentado que los descendientes prolongarán el legado familiar, de una generación a otra, de modo que la propiedad subsista indefinidamente. Esta sucesión trasmite la idea de eternidad al situarse más allá de la muerte de sus propietarios. Sin embargo, Henry, respondiendo a su mentalidad práctica americana, llega a casa con la voluntad de romper esa continuidad genealógica. Decide poner en venta todo su patrimonio familiar, sin importarle destruir su prolongación e ignorando que su madre aún habita Laxlinden. Con esta venta, Henry pretende liberarse de su pasado y la infancia traumática que ahí vivió, de la que culpa a Gerda. Henry nunca se sintió amado por una madre a la que acusa de desatenderle en favor de su marido y de su primogénito Sandy. La llegada de Henry a la casa y su espíritu destructor ocasionan un fuerte caos en la vida de Laxlinden, desafiando la estabilidad y el futuro de Gerda.

Cuando Henry vuelve a entrar en su dormitorio después de tantos años, experimenta un viaje en el tiempo. Su infancia se contempla como un periodo fosilizado, conservado en naftalina en su habitación. El museo de su infancia se conserva como si el tiempo se hubiera quedado suspendido en el momento que la desocupó. Henry la percibe como un sepulcro de su yo más joven que quedó ahí dormido y al que no pretende despertar:

The room was stripped to its minimum as if in an attempt, without destroying it, to make it forget. But it remembered. [...] All his old clothes were here, carefully hung in the wardrobe, carefully folded in the drawers, smelling of mothballs. A Henry museum. A Henry mausoleum. [...] It was all significant yet impersonal, archaeological [...] The room was old, waiting for a Henry who would certainly never return, who did not exist anymore. (MURDOCH, 1976: 85)

Si la casa funciona como soporte de su memoria, la puerta de acceso psicológico a la infancia de Henry se encuentra en la entrada al invernadero, por lo que «as he approached it now his heart beat suddenly with old undeclared memories» (Murdoch, 1976: 212). Como 
todos los espacios de Laxlinden, el invernadero también sufre las consecuencias del paso del tiempo, aunque algunas plantas aún resisten y sobreviven el paso de los años:

Its glory had now mainly departed, the heating system was no more, the decorative tiles were cracked or missing and much of the glass was broken at one end. Many of the plants and trees, put there by Henry's grandfather and great grandfather survived, however. (MURDOCH, 1976: 212)

El invernadero, que atesora plantas que ya fueron plantadas por el bisabuelo de Henry, pertenecería a la categoría que la socióloga Violette Morin denomina objetos biográficos (1969: 131), que incluye también lugares que poseen un fuerte componente identitario. Los olores que de él emanan estimulan en Henry la rememoración de recuerdos, despertando sensaciones dolorosas que le reconectan con su solitaria e infeliz infancia y con su hermano Sandy. Los diferentes aromas se mezclan y producen un desdoblamiento de su identidad: su yo actual y su Otro más joven: «a particular smell among the others declared itself, animating a much earlier Henry» (Murdoch, 1976: 85). Como lugar de preservación por excelencia, el invernadero sigue conservando su esencia, representando la continuidad que ha ido trasmitiéndose a lo largo de generaciones de Marshalson.

La propiedad familiar que Gerda se ha afanado en mantener inalterable, reteniendo objetos y tradiciones durante generaciones, comienza a experimentar cambios a causa del desapego material de Henry. Ahora es el joven amo quien controla sus pertenencias haciéndolas desaparecer a su antojo: "After all, you did things your way in your own time - and now it's my turn» (MuRDOCH, 1976: 224). La percepción de su casa como un fantasma supone el hundimiento de la propia Gerda, que siente cómo se desvanece lo que ha sido su vida hasta ese momento:

The house had changed. It had lived with Burke's life and with Sandy's life, and before Burke and before Sandy, it had cast its ray upon Gerda's childhood. Living nearby, she had loved the house before she had loved her husband; and when she came to it from her humbler home, as a bride of nineteen it had seemed a symbol of eternity. The house had been her education and her profession, and the men, Burke's widowed father, Burke, Sandy, had made it her shrine. But now, quite suddenly and unexpectedly, she and the house were strangers. (MURDOCH, 1976: 36)

\section{GERDA COMO VOZ EXCLUIDA}

Como categoría sociocultural, la edad representa un principio de organización social, que se refleja principalmente en la pertenencia a grupos de referencia, en las relaciones de poder que se establecen entre ellos y más concretamente en el conflicto generacional. Juventud y vejez emergen como un sistema binario y jerárquico, dando origen a prejuicios edadistas y a estereotipos discriminatorios. Pese a haber formado parte durante varios años de la vida social y cultural estadounidense, mucho más plural, liberada y moderna que su círculo británico, los prejuicios edadistas están arraigados en Henry. Su pragmatismo e in- 
dividualismo son notorios en el desprecio con el que trata a su madre por su edad, excluyéndola de todos sus planes: «After all, she's old now, she's had her life. She's wearing herself out trying to keep that ridiculous place going, she's always working in the house or the garden. It's about time she retired and sat still» (Murdoch, 1976: 177).

Las relaciones de poder que conlleva el envejecimiento se hacen patentes en el momento en que Henry invierte roles y ejerce su autoridad. Desempeñando el papel del opresor patriarcal, el joven Henry pretende controlar la vida de su madre, desempoderándola y excluyéndola de sus planes. «The country lady», apelativo que emplea familiarmente para referirse a ella su vecino Forbes, siempre se ha dedicado activamente a los quehaceres de la hacienda, resistiéndose a las restricciones que le impone su edad. La visión edadista de Henry, asumiendo de manera natural la construcción social de la vejez como un momento de pasividad y retiro, refuerza estereotipos negativos en torno al envejecimiento que se mantienen en la sociedad actual. En la pretensión de Henry de forzar a su madre a desengancharse de su vida activa, la opinión de Gerda queda silenciada.

Henry expone a su amigo Cato su intención de desprenderse de todos sus bienes familiares y alterar la estructura social en el que vive su madre: «My mother's living in a sort of feudal dream world. It's all false, it's a lie, and I'm going to smash it up» (Murdoch, 1976: 179). El escenario que proyecta Henry es que su madre se traslade a vivir a una sencilla casa con jardín situada en un pueblecito cercano, Dimmerstone. El pueblo también ha pasado a ser propiedad de Henry, y en su iglesia están enterrados todos sus antepasados. Henry espera que Gerda se adapte a una realidad que él mismo ha creado para ella: «She'll pick herself up and live to be the scourge of Dimmerstone» (Murdoch, 1976: 181). Así pues, con la edad Gerda no solo ve disminuida su renta, su poder y su belleza, sino también el espacio físico en el que va a vivir. A los ojos de Cato y del lector, el plan de Henry está proyectado para arruinar la vida de su madre, haciéndola morir de vergüenza. Este concepto simplista de su madre envejeciendo tranquila en una casita responde al arquetipo de la vejez como retiro en el hogar, pasividad en el interior de la casa y deterioro (WAXMAN, 1990: 10). Como sugiere Henry: «most elderly people have to accept some diminution in their lives. His mother was old enough for such a change and certainly young enough not to be slaughtered by it» (Murdoch, 1976: 235). Para Henry la mudanza a una casa más humilde descubrirá a su madre nuevas posibilidades para disfrutar de la vida, obligándola a adoptar el comportamiento sumiso que se espera de ella por su condición de persona mayor, adaptándose dócilmente a una nueva vida sin que ello la afecte, como si la vejez también hubiera disminuido sus sentimientos.

Esta visión de Henry responde a la tendencia a simplificar la vida que muestran generalmente las personas mayores (SHIELD y ARONSON, 2003), mudándose de grandes viviendas a pisos pequeños y retirándose de los actos sociales. Es la visión del mundo que se contrae: las cosas se vuelven más pequeñas y las necesidades más modestas. Sin embargo, en este caso no es Gerda quien escoge este cambio, sino que le viene impuesto por su hijo. La perspectiva de su compañero, Lucius Lamb, discurre en una dirección contraria. En oposición al modelo afirmativo del envejecimiento que encarna el personaje de Gerda, Lucius Lamb es un claro representante de la actitud tradicional frente al envejecimiento asociada exclusivamente al deterioro físico y mental, una muestra de las «decline narratives» frente 
a las «progress narratives» a las que alude Gullette (2004: 17). Al contrario que Gerda, Lucius vive su envejecimiento como una condición patológica, un modelo que Harry R. Moody asigna a la categorización de «ill-derly» frente a la de «well-derly» (en WorSFOLD, 2011: 183). Por lo tanto, Lucius acepta el estereotipo que Henry pretende imponer a su madre y a él; considera natural y beneficioso la desvinculación de las personas mayores de la sociedad:

We are getting old, my dear, you and I. If we lived in the east, we would be thinking of entering a monastery. Perhaps we ought really to give up the world. In a way, Henry is just making us do what we ought to do. We should live more simply at our age. (MURDOCH, 1976: 281)

Gerda sufre un duro golpe al verse desautorizada por su propio hijo y conocer el plan de vida que Henry proyecta para ella. En un primer momento, Gerda accede a sus peticiones sin atreverse a contradecirle. Sintiéndose desempoderada por él, accede a exiliarse en Dimmerstone y empezar de cero. La activación de etiquetas negativas de mujer mayor es un proceso que promueve la modificación de su propia conducta. Este comportamiento indica que Gerda ha interiorizado y adoptado el estereotipo cultural asumido que le impone su hijo. De esta forma, Gerda construye una identidad de acuerdo con lo que su hijo espera de ella y está dispuesta a desarrollar nuevos intereses para lograr congraciarse con él: «And let me tell you that the sooner I can retire and get rid of the responsibility of running this house the better I shall be pleased» (Murdoch, 1976: 219). Su estrategia es puramente adaptativa a las restricciones impuestas y a su actual condición de inferioridad, asumiendo una actuación tipificada, como sugiere su respuesta a su hijo: «what I think is not important. I am old» (MURDOCH, 1976: 253). Esta desvalorización la lleva a sentir que su vida ha concluido y sólo desea aislarse del mundo hasta entonces y esperar el final de sus días en soledad:

[A]ll she wanted now was to hide the scandal and the shame of it, the shame of being old and mad with misery and having lost her son. She would hide herself in the cottage at Dimmerstone and shut the door on the world forever. (MURDOCH, 1976: 254)

Como señala Sharon Kaufman, las personas mayores desarrollan un sentimiento de su propia identidad independiente de la edad, manteniendo una continuidad a pesar de los cambios que experimentan (1986: 6). En ocasiones, Gerda se resiste a que Lucius la incluya en la categoría de vieja «as for being old, speak for yourself» (Murdoch, 1976: 281). Su rechazo deja patente que en la sociedad occidental, admitir que se es viejo implica la pérdida de ciertos privilegios y la pertenencia a un grupo etario socialmente devaluado (Calasanti y SLEvin, 2006: 12). A través del comportamiento de su hijo, Gerda percibe por primera vez que está envejeciendo, tal y como explica a Lucius: «he has made us old. Have I come to the end of being a busy active sensible woman, and am now to become a useless whining spiteful old hag?» (MuRdoch, 1976: 282). Los términos peyorativos y hostiles de vieja, bruja, inútil, llorona y rencorosa usados por la propia Gerda para calificar a la mujer mayor corroboran que social y culturalmente el envejecimiento de las mujeres está marcado con potentes imágenes mentales desmoralizantes (FrEIXAS, 2008: 43). Ante el sentimiento tan profundo de pérdida y discontinuidad, Gerda experimenta un miedo a la vejez que no puede compartir con nadie, aumentando así su sensación de soledad: 
She shuddered with fear, fear for herself, for her sanity, for her continued being. She thought of the awful desolation of old age and death, which she could share with no one. Tears welled up and filled her eyes and her throat and she lay back moaning upon the bed. (MURDOCH, 1976: 186)

\section{EMPODERAMIENTO Y RESILIENCIA}

Una de las sorpresas que depara la edad a Gerda es convertirse en una mujer autónoma capaz de tomar sus propias decisiones. Aun presentándose en un primer momento como una mujer constreñida en un mundo patriarcal y androcéntrico, sometida a su marido y a sus hijos, en esta nueva etapa de su vida recupera su propia identidad adquiriendo una nueva seguridad. Gerda no está dispuesta a perder la única familia que le queda. Por eso, haciendo gala de su papel de estratega, cambia de táctica para conseguir manejar los hilos de la vida de su hijo. Invierte hábilmente su papel de mujer sumisa y se resiste a las expectativas que su hijo Henry le impone de acuerdo con un estereotipo de vejez, trasgrediendo sus esquemas de comportamiento. Ante las adversidades y las limitaciones que le presenta ahora la vida, Gerda muestra gran capacidad de superarse, rehacerse y resistir las imposiciones sociales, respondiendo a ellas con la asertividad que a menudo le llega a la mujer al envejecer:

A man may do what he likes with his own [...] and I cannot complain since I am, as you say, old and have had my time, but I do not want to stay here and see what is done to this place which I love. When the house is sold my life here will be over and finished. I shall leave and not come back. (MURDOCH, 1976: 343)

Gracias a esta resiliencia, finalmente Gerda es capaz de empoderarse ante las exigencias de Henry, verbalizando sus sentimientos y negándose a trasladarse a la casita a la que su hijo pretende enviarla. En su lugar, decide dejar la vida rural y mudarse a un apartamento en Londres: «I'm too old for gardening, as you pointed out yourself» (MURDOCH, 1976: 327).

$\mathrm{El}$ acontecimiento que permite restituir el orden en la vida de los Marshalson es la conversación que mantienen madre e hijo, en la que Henry le reprocha a Gerda su falta de afecto durante su infancia, que provocó la tensión que aún existe entre ellos. A partir de esta confesión Gerda tendrá la oportunidad de abrir las compuertas de sus emociones y enfrentarse a la acusación de su hijo, retratándose ella misma como una víctima de las circunstancias del pasado y del dominio de su marido:

I kept on sacrificing my will to him, and I kept on thinking that I had come to the end of my will and the end of the sacrifice, but there was always something more that he wanted from me that was hard to give. He loved me and it was one sort of way of having a satisfactory marriage. But I couldn't deal with both you and him. (MURDOCH, 1976: 413)

El personaje de Gerda ejemplifica cómo el envejecimiento proporciona mayor autonomía y libertad a la mujer. Tras la resolución del conflicto con su hijo Henry y la lib- 
eración de su carga emocional, Gerda realiza un viaje interior que la lleva a crecer y madurar. Esta serenidad adquirida tiene que ver con la emergencia de los llamados «procesos ejecutivos» que describe Anna Freixas (1991: 72) al referirse a las mejoras psicológicas que experimenta la mujer tras la mediana edad cuando, al conocerse mejor, siente la necesidad de ser su «yo» verdadero. Si la imagen de Gerda ha estado siempre asociada al campo como «the grand country lady», su madurez representará el momento de cosechar los frutos de su vida anterior. Cuando pensaba que su vida perdía su sentido, se abre ante Gerda una nueva etapa en la que toma por primera vez las riendas, experimentando un cambio hacia una nueva identidad, definida por un sentimiento de continuidad pero también por los cambios en su vida. Germanine Greer alude a esta transformación al referirse al momento en que la mujer madura «can bring about a quest for identity unhindered by reproductive aspects and a path towards real autonomy and an authentic self» (en KRIEBERNEGG y MAIERHOFER, 2014: 189). Esta visión positiva que promueve Greer se enfrenta al enfoque social tradicionalmente aceptado que define esta etapa vital en términos de pérdida. Gerda responde así a la definición de envejecimiento exitoso como oportunidad de crecimiento en la que retoma el control de su vida y recupera su voz. Su éxito se basa en la superación de los conflictos pasados así como de su dependencia emocional.

La reconstrucción de esta nueva etapa implica invertir la dirección del modelo de feminidad. Hasta el momento, Gerda había respondido a la imagen de cuidadora doméstica que como mujer se le había atribuido socialmente. No sólo cuida de la casa sino también de las personas que la habitan: anteriormente su marido y sus hijos y ahora Lucius Lamb, quien funciona como «passive care recipient» (KRIEBERNEGG y MAIERHOFER, 2014: 22). Cuando finalmente Henry reconsidera su decisión y permite que su madre permanezca en Laxlinden, Gerda decide reencontrarse con ella misma en soledad y liberarse de la responsabilidad de su papel de «ser-para y de-los-otros» (LAGARDE, 1996: 60). Ahora siente la necesidad de estar sola y librarse de las ataduras que no la dejaban avanzar. Así pues, aleja de su vida a su compañero Lucius y afronta por primera vez una soledad elegida y bienvenida:

I do want to be alone at last and without witnesses. You belong to the past, Lucius, as far as I'm concerned you're a ghost. Oh you are so stupid! You are stupid, so is Henry, so was Sandy, so was Burke. Oh God, why was my lot cast among such stupid stupid men! (MURDOCH, 1976: 408)

Frente al modelo de envejecimiento que encarna su amigo Lucius, que vive el envejecimiento como un proceso de declive y decadencia irreversible, Gerda sigue el modelo descrito por autoras como Sarton (2003) y Friedan (2006). Inesperadamente en esta etapa de su vida Gerda disfruta de menor tensión, menos conflictos y más libertad. A los ojos de Henry, su madre también recupera su dignidad. La integración de las identidades pasadas de Gerda proporciona a su hijo un sentimiento de continuidad y unidad: «Henry saw his mother, large and calm and ostentatiously bland, looking as he had so often seen her in the past. He saw that image going back and back as images in opposing mirrors» (MURDOCH, 1976: 381). En contra de lo que pensaba su hijo, Gerda sí dispone de un futuro y está decidida a no dejarlo pasar. La caída del tapiz polvoriento y pesado de finales del siglo XVII que pendía en la biblioteca de la mansión simboliza el paso a la modernidad. Con el tapiz se desvanece 
todo aquello que representa su vida pasada. Se abre ante Gerda un nuevo futuro que aparece como una pared fría y diáfana que lleva consigo una nueva visión de la biblioteca, el enigma ante un futuro que está por construir: «the library suddenly seemed much larger, much colder, as if the future had opened it up with rough chilling force» (MURDOCH, 1976: 277).

Autores como Laforest (1991) o Friedan (2006), enmarcan las relaciones sociales dentro de las necesidades básicas del ser humano a medida que se van cumpliendo años. Para redefinir su identidad, Gerda necesita abrirse al exterior, ampliar su círculo social y disfrutar de la compañía de otras personas. Su nueva etapa vital le ofrece la oportunidad de establecer nuevos vínculos y comenzar a cultivar la comunidad. Gerda olvida viejas rencillas vecinales y cruza los muros que la tienen enclaustrada en su hogar para encontrar apoyo emocional en John Forbes, quien comienza a visitarla a diario. Esta visión del amor maduro, entendido como una nueva forma de intimidad sin exigencias, produce en Gerda un efecto transformador que su hijo Henry ve reflejado en su aspecto físico rejuvenecido: «She looked much younger and had bought a lot of new clothes. [...] she was back again in the land of her youth, in the land proper to her own being. She too glowed» (Murdoch, 1976: 448).

La nueva etapa de la vida de Gerda y el cambio de rumbo de los planes de Henry coincide con la renovación de la mansión. La adaptación a la modernidad es visible en los objetos de la casa que recuperan el brillo de antaño. El tapiz de la biblioteca se rescata y vuelve a colgarse en su antiguo lugar, pero esta vez renace desprovisto del polvo del pasado y con una nueva luz y una inesperada tonalidad: «The bright light from outside showed up all its colours. Perhaps it had shed some of its dust or perhaps Gerda had never looked at it properly before [...] What she had vaguely thought of as black turned out to be a sort of marvellous indigo blue» (Murdoch, 1976: 434).

\section{CONCLUSIÓN}

Tomando como punto de partida la novela de Iris Murdoch Henry and Cato, el presente trabajo ha contribuido a comprender el modo en que se percibe culturalmente el envejecimiento en la sociedad contemporánea ateniéndonos a los cambios biológicos, psicológicos y sociales que conlleva este complejo proceso. A través del personaje de Gerda hemos analizado cómo la lectura de su cuerpo maduro la enfrenta al canon de belleza y (re)productividad encarnado por una generación más joven. Nuestro análisis también ha conectado el envejecimiento de Gerda con el deterioro de su casa, una mansión ancestral que también funciona como soporte de la memoria en el reencuentro de Henry con su pasado.

Del mismo modo también han quedado patentes otros factores sociales que conlleva la edad, puesto que en esta nueva etapa vital, Gerda no sólo se ha visto privada de su belleza, sino también de estatus y autoridad. Un cambio de roles asociados a estereotipos edadistas provoca que su hijo intente desempoderarla y excluirla de sus planes, obligándola a adoptar un comportamiento sumiso que espera de ella por su condición de persona mayor. Aunque Gerda en principio se adapta dócilmente siguiendo el modelo de envejecimiento que trata de imponerle Henry, finalmente logra resistirse. Así pues, hemos observado cómo Gerda 
responde a un modelo de envejecimiento que defiende una nueva generación de gerontólogos entendido como un proceso en el que la persona sigue creciendo y desarrollándose, sin tener por ello que negar su deterioro físico, y adaptándose con positividad a los cambios de la vida. Gerda ha llegado a un sitio nuevo y descubre la fuente de la edad que anunciaba Friedan, una visión opuesta al mito de la fuente cuyas aguas perpetuaban la eterna juventud. Esta visión no va ligada a la pérdida sino a la ganancia y acumulación (de años, experiencia y recuerdos) en la que coexisten valores como sabiduría, experiencia, sagacidad, tenacidad, fortaleza, perseverancia, resiliencia, resolución y determinación (SHIELD y ARONSON, 2003: 94). El envejecimiento para Gerda se representa como un momento gratificante de descubrimiento, de oportunidades y de liberación. Frey Waxman define esta nueva oportunidad de autorrealización como «literally tak[ing] the open road in search of [herself] and new roles in life» (1990: 16).

También Henry acabará aceptando que resulta inútil escapar del pasado, por lo que opta por dejar atrás el resentimiento y la venganza y perdonar a su madre. Al reconciliarse con su pasado, Henry y Gerda encuentran un punto medio, en el que el pasado (y Gerda) pueden tener aún un papel. Por eso Henry restablece el orden perturbado y vuelve a integrarse en la sociedad. El lector percibe que Henry ha concluido su peregrinaje moral desde la ceguera egoísta a la realidad. Un nuevo futuro se vislumbra tras este nuevo orden que se evidencia en su unión con Colette Forbes y en el anuncio de un hijo en común, el heredero que prolongará otra generación de Marshalson. La novela concluye en el momento en el que Henry sale de la casa y queda cegado por el potente sol del verano. Esta representación sugiere el símil platónico del sol a cuya luz es posible percibir la realidad, como imagen central del peregrinaje del ser humano. La relevancia de Murdoch como filósofa se ve reflejada en la alegoría de la caverna a la que a menudo recurre en su narrativa como metáfora de la transformación moral que sufren los personajes de sus novelas, quienes para convertirse en seres humanos moralmente mejores deben renunciar a su ego y abandonar su tendencia natural a habitar un mundo centrado en ilusiones y falsedad. Este peregrinaje espiritual hacia la realidad que existe fuera de uno mismo conduce al ser humano hacia la verdad y con ello hacia el bien.

\section{REFERENCIAS BIBLIOGRÁFICAS}

Arias, Rosario (2005): «Moments of Ageing: The Reifungsroman in Contemporary Fiction», Women Ageing through Literature and Experience, eds. Brian Worsfold and Grup Dedal-lit, Lleida, Edicions i Publicacions de la Universitat de Lleida, pp. 3-20.

Calasanti, Toni M. y Kathleen F. Slevin (2006): Age Matters: Realigning Feminist Thinking, New York, Routledge.

De Beauvoir, Simone (I996 (1970)): The Coming of Age, New York, Norton.

Deats, Sara Munson Y Lagretta Tallent Lenker (I999): Aging and Identity: A Humanities Perspective, Westport, Conn. [etc], Praeger,

Freixas, ANNA (I99I): «Autopercepción del proceso de envejecimiento en la mujer entre 50 y 60 años», Anuario de psicología, no ${ }^{\circ}$ 50, pp. 67-78.

Friedan, BetTy (2006): Fountain of Age, New York, Simon \& Schuster.

Gullette, Margaret Morganroth (2004): Aged by Culture, Chicago, University of Chicago Press. 
Hepworth, Mike (2000): Stories of Ageing, Buckingham, Philadelphia, Pa, Open University.

Hoskins, Janet (1998): Biographical Objects: How Things Tell the Stories of People's Lives, New York, Routledge.

Kaufman, Sharon R. (1986): The Ageless Self: Sources of Meaning in Late Life. Madison, Wis., University of Wisconsin Press.

Kriebernegg, Ulla y Roberta Maierhofer (20I4): The Ages of Life: Living and Aging in Conflict?, vol. 3, Bielefeld, Transcript Verlag.

LAFOREST, JACQUES (I99I): Introducción a la Gerontología: El Arte de Envejecer, Barcelona, Editorial Herder.

LaGarde, Marcela (1996): Género y feminismo: desarrollo humano y democracia, Madrid, Horas y horas.

Morin, Violette (i969) : «L'objet biographique», Communications, vol. 13, no 1, pp. 131-9.

Murdoch, IrIS (1976): Henry and Cato, London, Vintage Classics [versión Kindle].

SARTON, MAY (2003): «Toward another Dimension», Images of Aging: Cultural Representations of Later Life, eds. Mike Featherstone y Andrew Wernick, London [etc], Routledge, pp. 229-231.

Shield, Renée Rose y Stanley M. Aronson (2003): Aging in Today's World: Conversations between an Anthropologist and a Physician, New York, Berghahn Books.

Sodre, Ignes (1976): Introducción a Henry and Cato de Iris Murdoch, London, Vintage Classics [versión Kindle].

Sontag, Susan (1997): «The Double Standard of Aging», The Other within Us: Feminist Explorations of Women and Aging, ed. Marilyn Pearsall, Boulder, Colo., Westview Press, pp. 19-24.

Waxman, Barbara Frey (1990): From the Hearth to the Open Road: A Feminist Study of Aging in Contemporary Literature, vol. 113, New York [etc.], Greenwood.

Woodward, Kathleen M. (1991): Aging and its Discontents Freud and Other Fictions, Bloomington, Indiana University Press.

WorSFOLd, BRIAn J. (2011): Acculturating Age: Approaches to Cultural Gerontology, Lleida, Edicions i Publicacions de la Universitat de Lleida. 
